$\xi=-1$

\title{
Provide security for wireless mobile networks with different multiple access Techniques and MANET Network
}

\author{
E Uma Shankari ${ }^{1}$, S Rajashekhar Reddy ${ }^{2}$, P Aditya Sharma ${ }^{3}$, G Geetha ${ }^{4}$ \\ ${ }^{1,3,4}$ Department of Computer Science Engineering, Institute of Aeronautical Engineering, Hyderabad, India-500043 \\ ${ }^{2}$ Department of Computer Science Engineering, DRK College of Engineering and Technology, Hyderabad, India \\ *Corresponding author E-mail: umashankari.917@gmail.com
}

\begin{abstract}
The most recent versatile innovation of new features in mobile technology is needed for advancement of the Internet, for the features of better, quicker access of information. Cell division subscribers pay additional costs for instant messaging, stock quotes, and Furthermore easy internet access on their phones. In order to support such a capable system, we need pervasive, high-speed remote connectivity. Bluetooth and 802.11 are currently existing techniques for providing high speed digital wireless connectivity. The extension of $4 \mathrm{G}$ technology gave scope for mobile communications. Mobile is not only a portable talking gadget but also utilized for multiple distinctive purposes.4G offers higher data rates for consistent roaming. That portable mobile client might communicate without any disturbance due to network problems. In this process report we discuss different tests that $4 \mathrm{G}$ will facing and also gives the solutions for those problems. We recommend our own method for enhancing QoS On 4G eventually by utilizing both SMIP and SIP. We recommend that by utilizing such plan we might accomplish better QoS throughout that process of handover.
\end{abstract}

Keywords: Mobile Ad Hoc network, $4^{\text {th }}$ and $3^{\text {rd }}$ generation CDMA, MIMO, Quality of service

\section{Introduction}

The Growth and utilization of wireless networks has become need for increasing the expanded security identification. In this paper, we investigate the developments of remote security and identify open security issues connected with trust, management, interoperation and the problems, whose results need to aid separate over nature and friends. Wired networks which are made from network to network are completely misused by technology so for that we check possibility for remote systems administration. This will empower the further growth and also the speculation in remote systems administration. The innovation of these new features by provisions provides a new research for discriminating the dependable remote networks to provisional remote wireless networks. The establishment of wireless network provides the security for the given connections. We concentrate on different issues interrelated to different people concentrate on getting quick and secured portable network in order to avoid the traffic issues. Concerning the issue we change their cell division networks with 4G/LTE and at the same time including little phones and Wi-Fi right to support the ability and expansion scope. Wireless network determines that importance of the issues and the range for workable for providing a methodology in secure networks. When we think about the issues of designers include versatile specially appointed networks, open wireless networks for example, such as hotspot and mobile networks, including sensor networks.

\section{Securing Mobile Networks}

With an extending mobile wireless network system a new association focused includes every day versatile administrators which require a superior method to shield their system from a developing and running security dangers. By concocting a security technique that fragments the entrance systems (both cell and Wi-Fi) from the center system through expansion of an upgraded security gateway (SeGW), versatile administrators can better shield both themselves and their clients from hacking and assaults. The upgraded SeGW ought to give the accompanying security prerequisites. Tunneling the IP security improves SeGW groups with validation and exemplification techniques. Furthermore, for IP security (IPSec), the SeGW must provide encryption capacities including the new access associations from little cells and Wi-Fi to get focus on private system. VPN associations with big business and cloud systems. Burrowing gives a safe association into the versatile administrator's system from access and cloud organizes that they don't claim.

\subsection{Integrated firewall}

The improved SeGW should likewise give coordinated firewall capacities to shield the versatile administrator's system from getting the organized DoS assaults. OAM and control/flagging are debilitated by different assaults like flooding, twisted pairs, and ports examines. Since versatile administrators will convey distinc- 
tive portable system security models to ensure their 4G/LTE arrangement, the SeGW ought to be adaptable and bolster these diverse activity writes in either the same or diverse IPSec burrows alongside 1 or a few IKEv2 security affiliations.

\subsection{Self-organizing IPSec plug-n-play}

One of the key characteristics of 4G/LTE systems is simply the capacity for composing to move autonomously or naturally without administrator mediation. Radio access arrangement sellers give this self-sorting out system (SON) including the empowerment of recently introduced eNodeB to consequently set up an IPSec burrow for the OAM connect to its component administration framework. Improved SeGW bolsters all the fundamental portable system security capacities, for example, IKEv2 arrangement payload, multi-level testament confirmations, AAA server, DHCP and IP pool administration to help the IPSec plug-n-play methodology.

\subsection{High availability/network resiliency}

As portable administrators extend their impression and make utilization of unlicensed range, for example, LTE-U and Wi-Fi has limited client execution for critical increment in entrusted to get systems that requires more prominent IPSec scaling on the SeGW. Extra little cells and Wi-Fi get to focuses in the versatile administrator's system which include a request of extent of associations or more deeply - past the necessities of the large scale/metro cell arrange. With an expansive number of passage associations on the SeGW, it will be basic for it to be exceptionally versatile to equipment and framework disappointments. High-accessibility highlights, for example, state full multi-case flexibility can limit activity misfortune and counteract flagging.

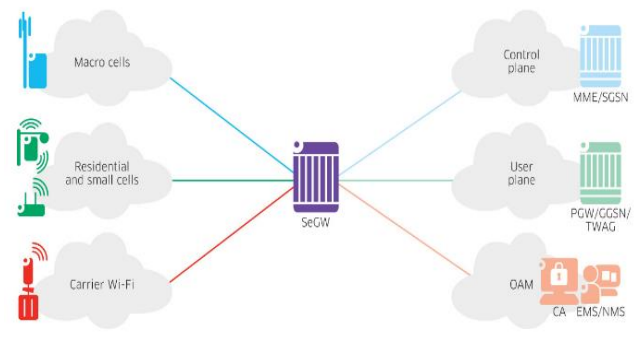

Figure.1. 4G/LTE and Wi-Fi services to protect their network and subscribers.

\section{Mobile generations}

\subsection{G applications}

3G can give acceptable higher data rates in both portable and fixed situations. It provides upon $2 \mathrm{Mbps}$ for stationary and something like $384 \mathrm{Kbps}$ for versatile environments. 3G needs supported video streaming and IP telephony and give less expense for mobile clients. $3 \mathrm{G}$ is the ITU standard to represent third era portable phone framework under that scope international mobile telecommunication system (IMT2000). 3G can execute through various networks like UMTS, GSM, CDMA, WCDMA, CDMA200, TDMA What's more edge.

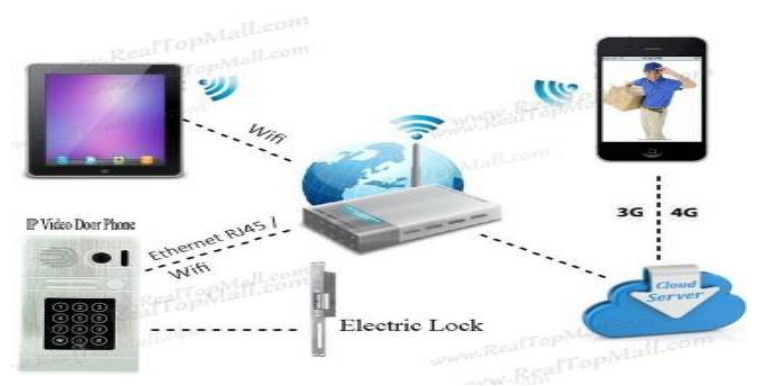

Figure.2. WIFI/3G/4G IP Video Door Phone IP Intercom System with Smartphone

\subsection{G Fourth generation}

Fourth generation (4G) likewise called Next Generation Network (NGN) offers one platform for various remote systems. These systems are associated through one IP center. 4G coordinates the current heterogeneous remote advancements keeping away from the need of new uniform standard for various remote frameworks like World Wide Interoperability for Microwave Access (WiMAX), Universal Mobile Telecommunications System (UMTS), remote neighborhood (WLAN) and General Packet Radio Service (GPRS). 4G systems will build the information rates staggeringly, by giving $100 \mathrm{Mbps}$ to $1 \mathrm{Gbps}$ individually

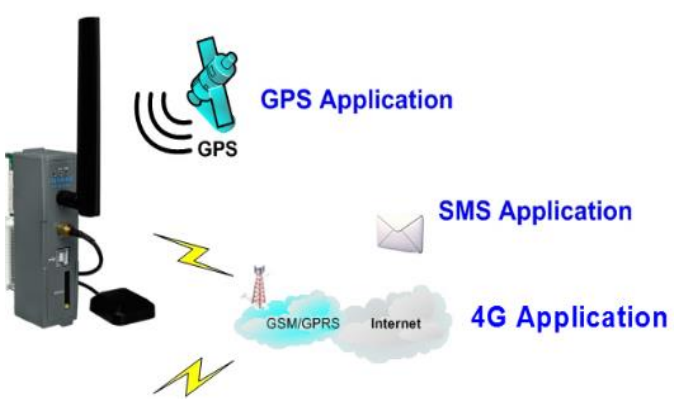

Figure.3. 4G Network

With the expansion in the information rates, the cell phones are made to perform higher execution applications. One such application in $4 \mathrm{G}$ is setting mindfulness. For instance if the versatile client is going by an office where he/she is having an arrangement to meet somebody and they have overlooked the arrangement. On the off chance that the workplace area, address and topographical area coordinates the one client has just put away in the telephone, he/she will get data about the arrangement and will be reminded that you have to play out this action.3.3 LTE Long Term Evaluation

LTE stands for Long term Evolution and it is used for accomplishing $4 \mathrm{G}$ speeds by technical innovations. The way things are, more often than not when your telephone shows the "4G" image in the upper right corner, it doesn't generally would not joke about this. The point when those ITU-R situated to build velocities for $4 \mathrm{G}$, they were to some degree inaccessible, despite those measure of money geek makers place under accomplishing them. Accordingly, the running muscle to picked that LTE, those name provided for of the advancement used Likewise a and only mission to the individuals norms, Might be denoted Concerning illustration $4 \mathrm{G}$ in the off chance that it provided for An liberal change over the $3 \mathrm{G}$ advancement. Quickly arranges started promoting their associations as $4 \mathrm{G}$ LTE, a showcasing procedure that enabled them to assert cutting edge network without reaching the genuine required number first; it would resemble the U.S. asserting they had arrived on the moon since they got really close and the spaceship that got them there was a great deal superior to the past ship. It's not so 
much cunning however, in spite of conflicting rates relying upon area and system, the contrast in the vicinity of $3 \mathrm{G}$ and $4 \mathrm{G}$ is quickly observable.

On the genuine inquiry is, would you be able to feel a contrast in the vicinity of $4 \mathrm{G}$ and LTE systems? Is the speed of stacking a page or downloading an application on your handheld gadget a great deal quicker in the event that you have LTE innovation inherent? Most likely not, unless you live in a city. While the contrast between slower $3 \mathrm{G}$ systems and new $4 \mathrm{G}$ or LTE systems is unquestionably extremely detectable, a significant number of the $4 \mathrm{G}$ and "genuine $4 \mathrm{G}$ " systems have transfer and download speeds that are relatively indistinguishable. The take-off of LTE-An is beginning to have any kind of effect, yet your mileage may fluctuate. For the time being, LTE-An is the speediest association accessible for remote systems.

\section{Developing with networks}

In spite of the fact that there would distinctive ideas towards $4 \mathrm{G}$, network components every now and again come up as a supporting and solving critical solutions that help to achieve in advancement towards $4 \mathrm{G}$. In this session we need to aid research and demonstrate technical innovations for example, MIMO (Multiple-Input Multiple-Output), OFDMA (Orthogonal frequency division multiple Access) that Might fundamentally build security, versatility and throughput about $4 \mathrm{G}$.

\subsection{MIMO}

Envision that you could listen finer what you need to listen, Furthermore don't listen the thing that bothers you, simply by pointing out the place message What's more noises would advancing. From shaft shaping that is those noteworthy idea of MIMO (Multiple-Input Multiple-Output) permits you do barely that utilizing advanced mobile antennas framework. Expanding pace Furthermore range, MIMO is at that point acknowledged by analysts Similarly as a standout amongst the fundamental parts about undertakings for example, such that WiBro, WiMAX, WLAN, 802. 11n, UMTS R8 LTE, Further more UMB.

\subsection{OFDMA Evolution}

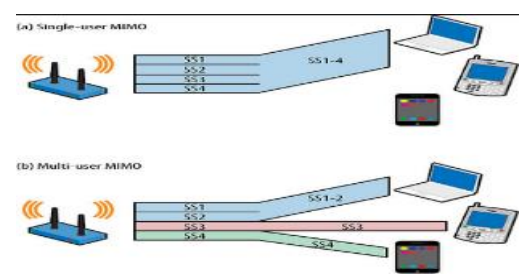

Figure.4. MIMO network

A normal investigate organization recommended the simplest route to execute MIMO by utilizing the frequency of OFDM, that together fundamentally can expand the execution eventually to a extending range, boosting speed and also enhancing the quality. OFDM will adjust and isolate the allocated recurrence channel into number of limited gropus by limiting the independency of subcarriers for the limited objective that there may be no impedance between them; signals need to aid orthogonal.

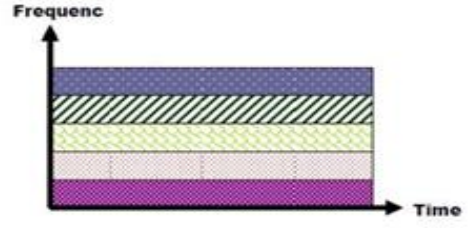

Figure.4.1. OFDM Model

Before OFDM wind up prevalent, three different arrangements were exhibited to share radio range between numerous clients. Utilized by 1G, FDMA :parcel the channel by users.TDMA it was suggested that allot every client access to the entire transmission capacity for brief timeframe

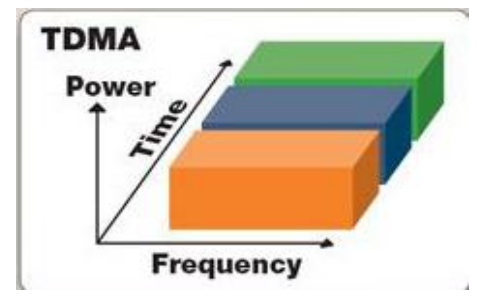

Figure. 4.2

Since TDMA in $2 \mathrm{G}$ caused obstruction issue, CDMA was proposed as another type of multiplexing where every client was permitted to utilize the entire channel limit

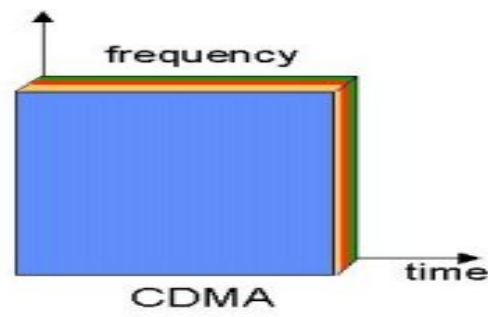

Figure.4.3 CDMA Model

The better information throughput end up conceivable, when utilizing orthogonal recurrence division multiplexing, the radio data transfer capacity could be subdivided into limit groups.
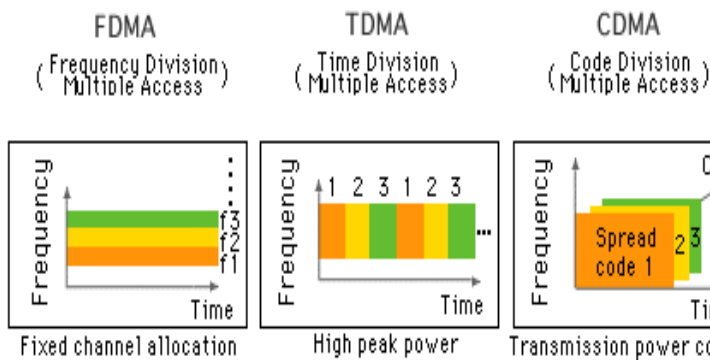

Figure.4.4. Multiplexing Techniques

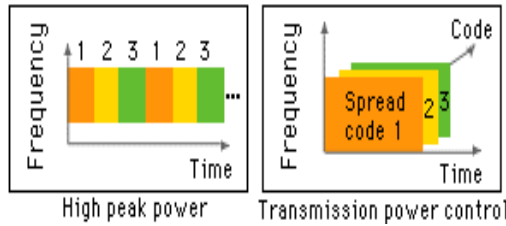

\subsection{Mobile AD HOC Networks}

Specially appointed systems, for example, Bluetooth are systems intended to progressively interface remote gadgets, for example, mobile phones, PCs, and PDAs. These systems are named "impromptu" on account of their moving system topologies. While WLANs utilize a settled system framework, impromptu systems keep up irregular system setups, depending on an ace slave framework associated by remote connects to empower gadgets to impart. In a Bluetooth organize, the ace of the piconet controls the changing system topologies of these systems. It additionally controls the stream of information between gadgets that are equipped for supporting direct connects to each other. As gadgets move about in a capricious manner, these systems must be reconfigured 
on the travel to deal with the dynamic topology. The steering that convention Bluetooth utilizes enables the ace to set up and keep up these moving systems.

\section{Mobile Security}

Security to advanced reality implies should secure the advanced frameworks starting with criminal and unapproved utilization. As far as machines What's more portable correspondences the necessity for security need expanded overwhelmingly for the change previously, innovation. A percentage decades back at main era of portable networks were being used the idea for security might have been not to such an extent to act or we could say that mindfulness might have been not that significantly highlighted. Be that as Likewise engineering organization held ahead enhancing What's more new advents were presented the require about security kept ahead crawling. These times nobody loves will a chance to be unstable digitally. Due to the overwhelming reliance looking into advanced networking for the utilization from claiming private, sensitive, fiscal and significant correspondence. There might make numerous strike for advanced information a few for them would eavesdropping, mamoncillo in the center attack, refusal of administration (DOS) attack, spoofing What's more part more.

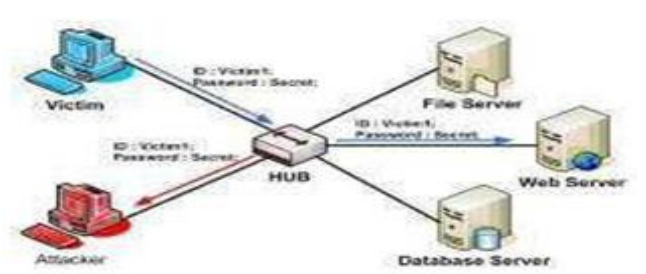

Figure 5.1. Eavesdropping

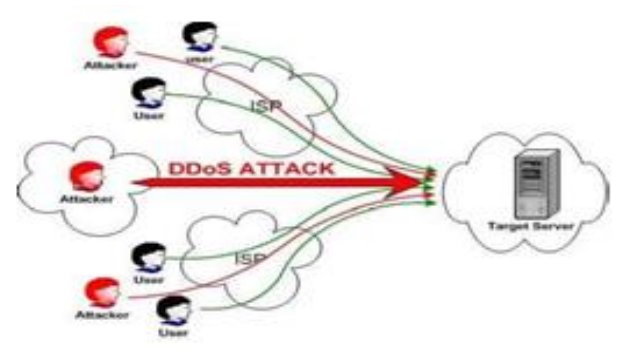

Figure 5.2. Denial of Service

Traditionally network security is considered to secure network edges from external attacks Shockingly that this is not addition Similarly as attackers search for breaches for network protocols, operating systems and more applications. Consequently we require a security instrument that could ensure the entirety system

\section{Quality of Service In $4 \mathrm{~g}$}

In media transmission the term QoS (Quality of Service) remains for the asset reservation control instrument, rather than the interpretation of term as accomplished administration quality. Correspondence happens when the information streams from source to goal and QoS ensures a predefined level of bit rate, jitter, and deferral and bundle drop likelihood to the stream. QoS confirmation is critical for constant traffics like Voice over IP (VoIP), web based gaming, IP TV and video gushing and so on. QoS empowers arrange executives to maintain a strategic distance from organize blockage and deal with the system assets proficiently.

The goal of the $4 \mathrm{G}$ is to provide the users the facility of Always Best Connected (ABC concept). To provide QoS in $4 \mathrm{G}$ is not simple and easy job as one has to deal with different parameters in different technologies. Like if a user is moving and changing his coverage network, so to provide service under QoS framework is challenging.

There are a few conventions intended to keep up the consistent correspondence of the clients while moving or as it were to limit the idleness and bundle loss of the progressing correspondence session. The versatility conventions are Mobile IPv6, Hierarchical MIPv6, Fast MIPv6.These conventions can help in enhancing the portability administration of versatile clients. Keeping in mind the end goal to give QoS to the versatile clients we propose a blend of portability convention Seamless Mobile IPv6 (SMIPv6) and Session Imitation Protocol (SIP). Session Initiation Protocol (SIP) is utilized to oversee portability of various elements, for example, session, terminal, administration and individual versatility. It encourages versatility and keeps up the ongoing sight and sound sessions. Taste is an application layer convention thusly it can work both in IPv4 and IPv6.

\section{Conclusions}

In this paper we are describing about the various wireless mobile technologies, and various applications of $4 \mathrm{G}$ mobile communication as well as the LTE (Long Term Evolution). And also we describe about various networks we are used in 4G, such as MIMO and OFDMA Evolution, in that we discus about FDMA, CDMA, as well as TDMA. And also describes the Security, Quality of Service in $4 \mathrm{G}$. We present the challenges that $4 \mathrm{G}$ faces and their up-to-date solutions. To enhance the QoS in $4 \mathrm{G}$ we propose our own particular plan of consolidating versatility convention SMIP and application layer convention SIP. We can ensure the asset distribution amid the handover procedure by consolidating the two conventions and versatility administration can be upgraded.

\section{References}

[1] M. King and B. Zhu, "Gaming strategies," in Path Planning to the West, vol. II, S. Tang and M. King, Eds. Xian: Jiaoda Press, 1998, pp. 158-176.

[2] B. Simpson, et al, "Title of paper goes here if known," unpublished.

[3] J.-G. Lu, "Title of paper with only the first word capitalized," $J$. Name Stand. Abbrev., in press.

[4] M. Young, The Technical Writer's Handbook, Mill Valley, CA: University Science, 1989.

[5] Mitchell Ashley "A Guide to Wireless Network Security" Information systems Control Journal ,Volume 3,2004.

[6] Karen Scarfone, Derric Dicoi, "Wireless Network Security for IEEE 802.11a/b/g,Bluetooth(DRAFT)",NISTPublication-80048.Augest 2007

[7] Tom karygiannis, Les Owens, "Wireless Network Security for IEEE $\quad 802.11 \mathrm{a} / \mathrm{b} / \mathrm{g}$, Bluetooth(DRAFT)",NISTPublication-80048.November 2002

[8] Ahmed M. Al Naamany, Ali Al Shidhani, Hadj Bourdoucen, "IEEE 802.11 Wireless LAN Security Overview", IJCSNS International Journal of Computer Science and Network Security, VOL.6 No.5B, May 2006.

[9] Omar Cheikhrouhou \& Maryline Laurent \& Amin Ben Abdallah \& Maher Ben Jemaa, "An EAP-EHash authentication method adapted to resource co 